\title{
Proposal for Step-up Gait of RT-Mover, A Four-Wheel-Type Mobile Robot for Rough Terrain with Simple Leg Mechanism
}

\author{
Shuro Nakajima, Member, IEEE
}

\begin{abstract}
RT-Mover can move on continuous rough terrain using a motion control technique proposed in a previous paper[1]. There is, however, larger rough terrain in a real environment than in the previous paper, so, RT-Mover can not operate there using that technique. In this paper, a step-up gait for an upward step is proposed for use in discontinuous rough terrain. The flow of processes in the step-up gait and stability during the gait are discussed. The proposed gait is evaluated through simulation and experiment.
\end{abstract}

\section{INTRODUCTION}

There is a strong demand for mobile robots that can move on rough terrain in various applications, for example, to aid people who have difficulty in walking. However, few robots are suitable for use in rough terrain at practical level.

Many rough terrain mobile robots at research level exist. Most of them are classified into the following three categories.

1) Legged robots: These have excellent mobility with high stability; legs are used to support the body and maintain its stability. The mobility of legged robots has been extensively studied, e.g. ASV[2] and the TITAN series[3].

2) Wheeled robots: These are most commonly selected for traversing continuous surfaces that include rough terrain. Because of their stability, maneuverability, and simple controls, wheel mechanism is the most frequently used for exploration rovers. Among examples of wheeled mobile robots, Micro5[4] and Rocky7[5] have passive linkage mechanisms, and SpaceCat[6] and Nanokhod[7] have active linkage mechanisms.

3) Wheeled legged robots: These have the merits of both legs and wheels. Work Partner[8] and Roller Walker[9] are equipped with wheels placed at the ends of the legs. Chariot III[10] and RoboTrac[11] have separate wheels and legs.

Although a legged mechanism is highly mobile on rough terrain, it is complex and more energy is required for walking. On the other hand, most of wheeled robots can not travel over discontinuous terrain; however, they are usually the best solution for continuous terrain. A hybrid mechanism provides the strengths of both wheels and legs, although such mechanisms also tend to be complex.

RT-Mover[1] has a simple mechanism and enough mobility for the following target environments: 1 . An indoor environment with an uneven ground surface, 2. An artificial outdoor environment with an uneven ground surface and a

This work was partially supported by KAKENHI (19700194, Grant-inAid for Young Scientists (B)).

Shuro Nakajima is with the Department of Advanced Robotics, Chiba Institute of Technology, 2-17-1, Tsudanuma, Narashino, Chiba 275-0016, Japan. shuro.nakajima@it-chiba.ac.jp staircase, and 3. Natural terrain such as a trail in a forest. Its mechanism is different from those of conventional mobile robots. Four wheels are mounted at the tip of every leg, and the leg mechanism is quite simple (Fig.1). RT-Mover has four active wheels and only five active shafts, and it can move on discontinuous rough terrain while maintaining a sheet-like body horizontally. It can move like a wheeled robot and also walk over a step like a legged robot, despite the simplicity of the mechanism.

In this paper, a step-up gait for an upward step is proposed. The flow of processes in the step-up gait, its stability, and the kinematics during this gait are discussed. The proposed gait is then evaluated through simulation and experiment.

\section{RT-MOVER}

Fig.1 shows RT-Mover, the four-wheel-type mobile robot built for rough terrain with a simple leg mechanism that I discuss in this paper. It has four driving wheels, front and rear steering shafts, front and rear roll-adjustment shafts, and a seat pitch adjustment shaft at the center of its body. RTMover is equipped with sensors: an encoder and a current sensor for each joint motor, and posture angle sensors relative to its seat part (pitch and roll).

The robot is characterized by the smallest number of driving shafts that assist its movement on discontinuous rough terrain while maintaining its seat part in a horizontal plane, where an occupant or load is seated. Other than the wheels, it has only five degrees of freedom in total. In reference [1], I showed that RT-Mover can move on continuous rough terrain while maintaining the seat part in a horizontal plane by applying equation (1) to the pitch adjustment shaft and each front and rear roll-adjustment shaft (basic movement control method).

$$
T_{d}=K\left(\theta_{d}-\theta\right)+D\left(\dot{\theta}_{d}-\dot{\theta}\right)=-K \theta-D \dot{\theta},
$$

$T_{d}$ : target torque; $\theta$ : seat part's posture angle; $\theta_{d}$ : seat part' s target posture angle (=0); $K$ and $D$ : angle gain and angular velocity gain.

Using the control method in equation (1), RT-Mover can move on rough terrain while maintaining its posture and its wheels can be in continuous contact with the ground. However, with large steps or gaps, the ground contact points of the wheels need to be altered by lifting the wheels. In this study, I discuss a step-up moving method as the first step of studying a moving method on discontinuous rough terrain. I now consider a case of lifting a wheel onto a step on which the wheel cannot move (Fig.2). 


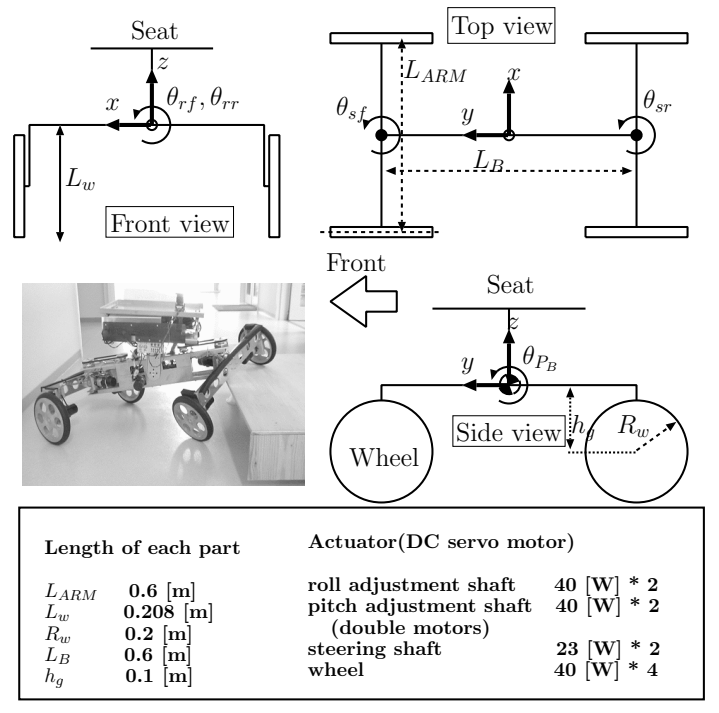

Fig. 1. The rough terrain mobile robot RT-Mover

Assuming that static stability is maintained during the movement, a wheel is lifted like a leg while constantly supporting the body on at least three points in order to position the center of gravity on a supporting polygon. Since the robot cannot move its center of gravity without altering the supporting points due to its small degree of freedom, the position of the supporting point is adjusted by rotating the steering shafts in order to maintain static stability. Of the three supporting points, since the steering shaft on the wheel-lifting side (leg-side steering) is used for moving the lifted wheel forward, static stability is increased by rotating the steering shaft of the other side (support-side steering) (for example, Fig.3(c)). Since the left-right order does not affect the movement in the step shown in Fig.2, the robot can move onto the step by lifting the wheels one by one in the order front-left, front-right, rear-left, and rear-right (Fig.3).

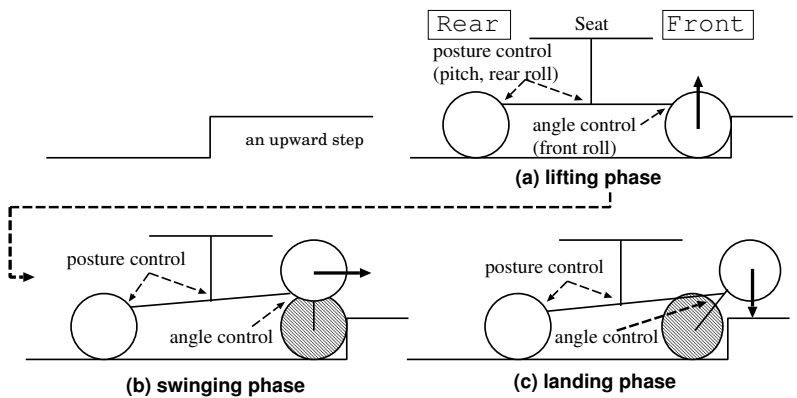

Fig. 2. An upward step

\section{Study of Stability}

I now study whether the robot can, while maintaining static stability, move over a step of $0.2[\mathrm{~m}]$ with the above gait strategy. Fig.4(a) shows the static stability when lifting a wheel. Static stability is seen if the center of gravity is in the supporting polygon. Since RT-Mover employs a mechanism with minimum number of driving shafts, it cannot move its center of gravity without altering the supporting points. In

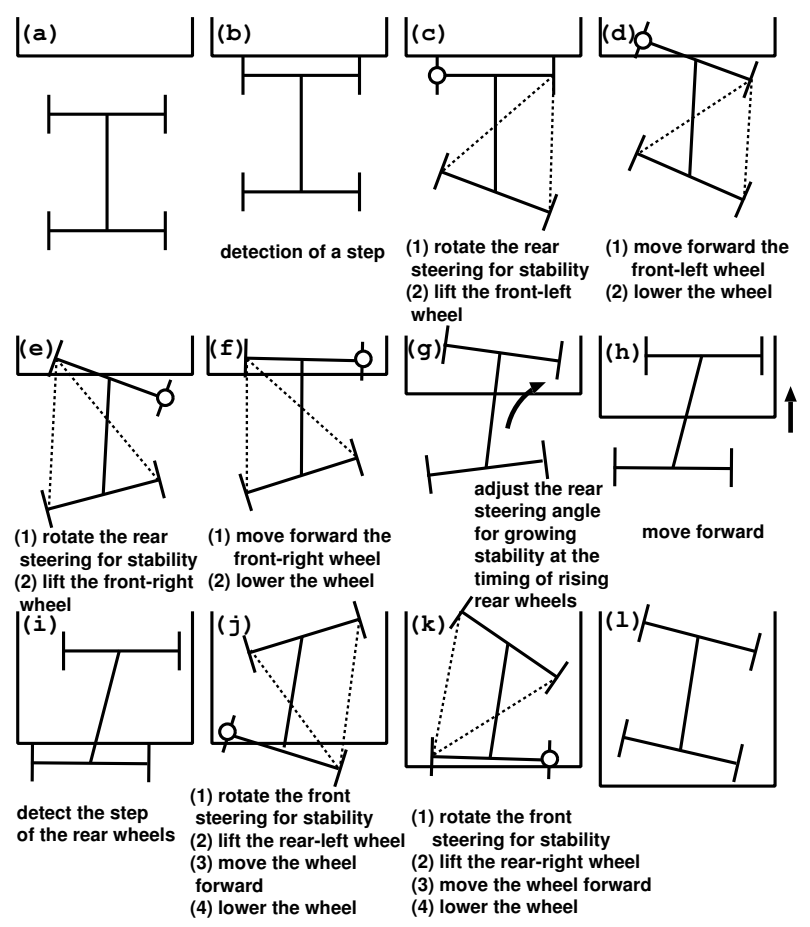

Fig. 3. Flow of processes in the step-up gait

addition, the supporting point of the front-right wheel in Fig.4(a) cannot move since it needs to move the lifted wheel forward. Thus, the rear steering is used so that the center of gravity stays within the supporting polygon.

As shown in Fig.4(b), if the body inclines backwards when going up a step, the center of gravity is displaced backwards by $h_{g} \sin \theta_{P_{B}} . \theta_{P_{B}}$ is the body pitch angle.

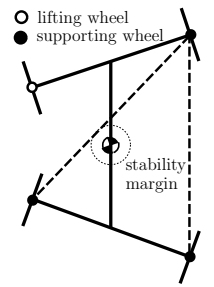

(a)

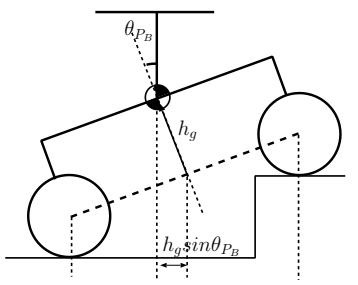

(b)
Fig. 4. Stability margin

Of the four scenarios of lifting each wheel during the stepup gait, lifting a rear wheel (Fig.5(c) and (d)) causes static instability since the center of gravity is displaced backwards due to backward inclination of the body. Thus, I discuss static stability shown in Fig.5(c) and (d). Here, the front wheels are rotated up to the limit of $\pm 30\left[^{\circ}\right]$ toward the direction that increases stability.

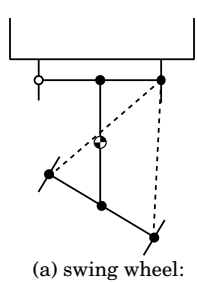
a) swing wheel
front-left

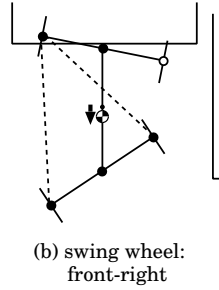

front-right

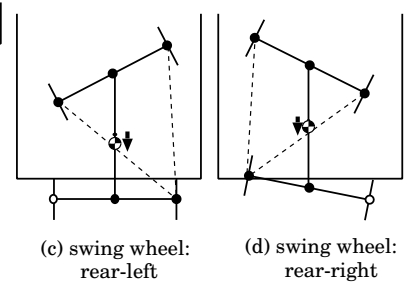

rear-left

Fig. 5. Four scenarios using the step-up gait 
The rear-left wheel is lifted, moved forward, and then lowered. In that position, the rear-right wheel is lifted, moved forward, and lowered; therefore the rear steering angle when the rear-right wheel is lifted depends on the rear steering angle when the rear-left wheel is lifted. It can be seen in Fig.5 that when lifting a rear wheel, the less the lifted wheel goes forward, the more the robot has static stability. Hence, I assume this advancement of the rear-left wheel to be the minimum required amount for going up the step. Since the wheel can be placed on the step by advancing the wheel at its radius from the state shown in Fig.5(c), I can assume the steering angle to move at $\sin ^{-1}\left(R_{w} / L_{A R M}\right)$. Also, since the rear-left wheel is already on the step when lifting the rear-right wheel, the inclination of the body posture angle is smaller in (d) than in (c).

By taking these into account, Fig.6 shows the result of calculation of static stability as the minimum distance between the center of gravity and the supporting polygon on a $0.2[\mathrm{~m}]$-high step. This data is approximation because of not considering $L_{w}$ in Fig. 7. $0.2[\mathrm{~m}]$ is the wheel diameter, and is the max height as which RT-Mover targets. The horizontal axis represents the rear steering angle when lifting the rearleft leg, and the vertical axis represents static stability. A positive value of static stability indicates good stability, and negative indicates instability. The solid line represents a change in static stability of the rear steering angle when lifting the rear-left leg, and the dotted line the rear-right leg. Fig.6 shows that it is possible to position both rear wheels onto the $0.2[\mathrm{~m}]$ step while maintaining static stability by setting a rear steering angle at -1 to $12\left[^{\circ}\right.$ ] when lifting the rear-left leg.

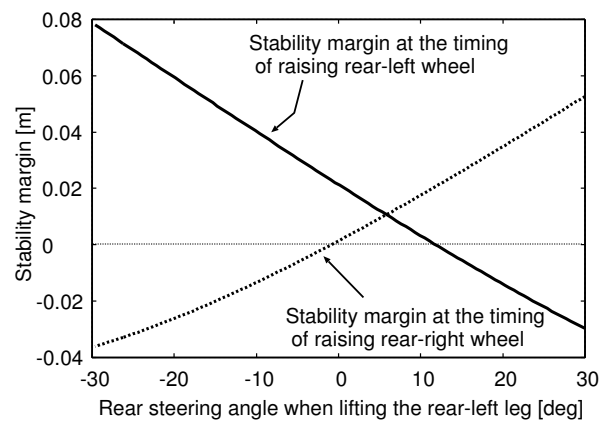

Fig. 6. Stability margin data V. STUDY OF TRAJECTORY

The kinematics of the step-up gait in detail will be presented in another report. Here I present only the summary. In the below analysis, I use a "projection frame" (Fig.7), which comprises projecting line segments connecting the wheel landing points (arms) and a line segment connecting the centers of the arms (body) to horizontal planes. I let the body center of the projection frame be the origin, the direction of movement be $\mathrm{Y}$, and vertically upward direction be $\mathrm{Z}$, and I use this right-handed system as the body coordinate system.

\section{A. Lifting and Landing Phase}

When lifting or landing the front-right wheel, a velocity command value will be set for the front roll-adjustment shaft.

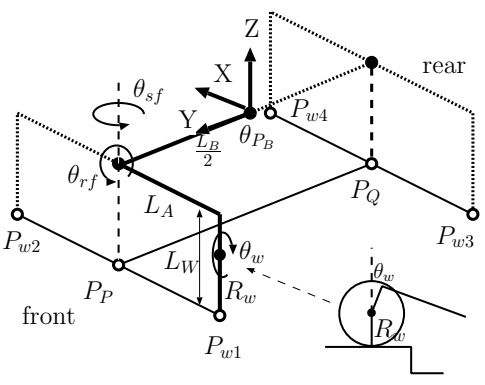

Fig. 7. Model for analysis

In order to avoid contacting the step lateral surface, the wheel is moved up and down without moving back or forth. As will be stated in the next chapter, the posture control in equation (1) is applied to pitch adjustment and rear roll-adjustment shafts, and rotation of the lifted front-right wheel and the supporting front-left wheel is stopped. In order to widen the supporting polygon, the rear steering shaft is rotated to its steering limit. The control parameters of the front steering shaft, the rear-left wheel, and the rear-right wheel are determined by the value set for the front roll-adjustment shaft.

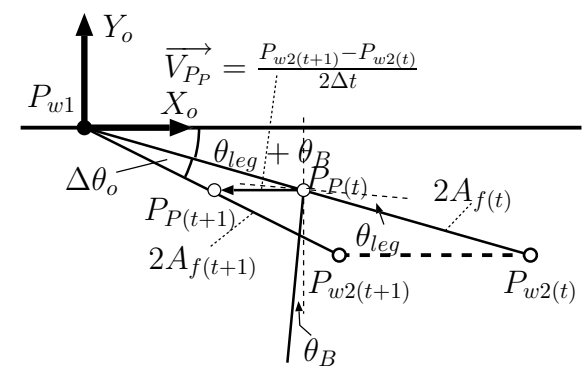

Fig. 8. Calculation model for trajectory of the leg when raising and lowering a wheel

Now I explain how to derive these control parameters. As shown in Fig.8, I discuss it on an absolute coordinate system with its origin at the landing position of the front-left wheel $\left(P_{w 1}\right)$. In Fig.8, I assume the position of the front-right wheel $\left(P_{w 2}\right)$ when moving the front roll-adjustment shaft for a small amount of time $\Delta t$, at a velocity of $P_{P}$ and a small angle $\Delta \theta_{o}$, and derive the angular velocity of the front steering shaft $\dot{\theta}_{S f}$ and the velocities of the rear-left and rear-right wheels $\overrightarrow{V_{w 3}}$ and $\overrightarrow{V_{w 4}}$. Since the wheel is moved up and down without moving in the $\mathrm{Y}$ direction, the $\mathrm{Y}$ coordinate of $P_{P}$ is constant.

$A_{f(t)}$ is the distance between $P_{w 1(t)}$ and $P_{P(t)}$, and it depends on the front steering $\theta_{s f}$, the front roll-adjustment shaft $\theta_{r f}$, and the body pitch angle $\theta_{P_{B}} . A_{f(t+1)}$ is $A_{f}$ when $\theta_{r f}$ is moving slightly. However, $\theta_{s f}$ and $\theta_{P_{B}}$ are approximated to those of time $t$ since it is difficult to assess them analytically.

I skip some steps and eventually obtain the velocity of point $P_{P}\left(\overrightarrow{V_{P_{P}}}\right)$ and $\Delta \theta_{o}$ :

$$
\begin{gathered}
\overrightarrow{V_{P P}}=\left(\frac{P_{w 2 x(t+1)}-P_{w 2 x(t)}}{2 \Delta t}, 0\right), \\
\Delta \theta_{o}=-\tan ^{-1} \frac{P_{w 2 y(t)}}{P_{w 2 x(t)}}-\tan ^{-1} \frac{P_{w 2 y(t+1)}}{P_{w 2 x(t+1)}} .
\end{gathered}
$$


Since $\Delta \theta_{o}$ includes the projected front steer angle $\theta_{l e g}$ and the body rotation $\theta_{B}$, I get

$$
\Delta \theta_{o}=\Delta \theta_{\text {leg }}+\Delta \theta_{B}
$$

Among these variables, the one that includes the control parameter of the front steering shaft is $\dot{\theta}_{\text {leg }}$, and the control parameter of the front steering is determined by calculating $\dot{\theta}_{B}$ and the relationship between $\dot{\theta}_{l e g}$ and $\dot{\theta}_{s f}$.

$\dot{\theta}_{s f}$ can be obtained by the below geometric relationship. $\theta_{P_{B}}$ is obtained from the information obtained by the posture angle sensor on the seat part and the angle of the pitch adjustment shaft.

$$
\theta_{l e g}=\theta_{s f} \cos \theta_{P_{B}}+\theta_{r f} \sin \theta_{P_{B}} .
$$

The velocity of the body rotation $\dot{\theta}_{B}$ is obtained as shown below. Here, $B$ is a projected body length and $V_{P_{Q x}}$ is derived from velocities of the rear-left and rear-right wheels, which will be discussed next.

$$
\dot{\theta}_{B}=\frac{V_{P_{Q x}}-V_{P_{P x}}}{B} .
$$

1) Control Amounts of Rear-Left and Rear-Right Wheels: In Fig.9, taking the velocities gained by point $P_{P}$ gains from the velocities of the rear wheels as $\overrightarrow{V_{P_{P 3}}}$ and $\overrightarrow{V_{P_{P 4}}}$, the velocity of $P_{P}$ is

$$
\overrightarrow{V_{P_{P}}}=\overrightarrow{V_{P_{P^{3}}}}+\overrightarrow{V_{P_{P w 4}}} \text {. }
$$

Moreover, since the body length $B$ changes as $\theta_{P_{B}}$ changes, as for $\overrightarrow{V_{w 3}}$ and $\overrightarrow{V_{w 4}}$, the amount of change $\left(\left(B_{(t+1)}-B_{(t)}\right) / \Delta t\right)$ must also be considered. Therefore, I get

$$
\begin{aligned}
& \left|\overrightarrow{V_{w 3}}\right|=\frac{2 A_{r}}{L R}\left|\overrightarrow{V_{P_{w w}}}\right|+\dot{B}, \\
& \left|\overrightarrow{V_{w 4}}\right|=\frac{2 A_{r}}{L L}\left|\overrightarrow{V_{P_{w 4}}}\right|+\dot{B} .
\end{aligned}
$$

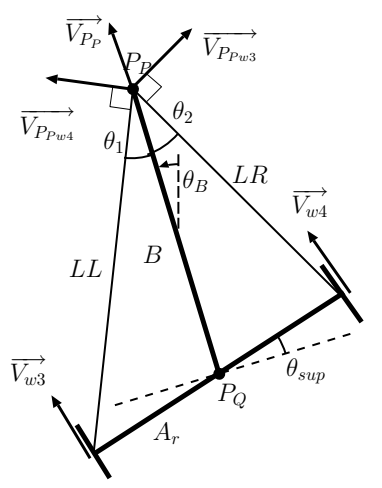

Fig. 9. Calculation model for $V_{w 3}$ and $V_{w 4}$

\section{B. Swing Phase}

Fig.10 is a model of the swing phase, wherein the origin of the absolute coordinate system is the front-left wheel and the lifted leg is represented by the front-right wheel. The trajectory is set such that point $P_{P}$ draws a circular path around the front-left wheel. Velocities of the front steering shaft and rear wheels are determined such that they satisfy

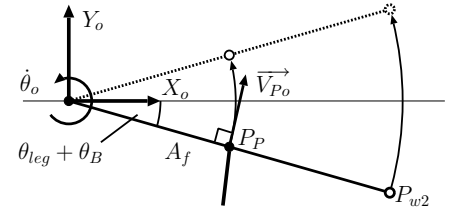

Fig. 10. Calculation model for the swing phase

$\overrightarrow{V_{P_{P}}}$. Letting $\overrightarrow{V_{P_{P}}}$ be the set value that gives the angular velocity $\dot{\theta}_{o}$, I obtain the below:

$$
\left|\overrightarrow{V_{P}}\right|=A_{f}\left|\dot{\theta}_{o}\right|
$$

$$
\overrightarrow{V_{P_{P}}}=\left(-\left|\overrightarrow{V_{P}}\right| \sin \left(\theta_{\text {leg }}+\theta_{B}\right),\left|\overrightarrow{V_{P_{P}}}\right| \cos \left(\theta_{\text {leg }}+\theta_{B}\right)\right) .
$$

With the velocity of point $P_{P}$ determined, as in the lifting and landing phases, the control parameter of the front steering adjustment shaft and velocities of the rear wheels can be obtained.

Fig.11 shows the position of the front-left wheel along the $\mathrm{Y}$ and $\mathrm{Z}$ axes when the initial position of the front steering $\theta_{s f}$ is 20 [ $^{\circ}$ ] and the rear steering $\theta_{s r}$ is -30 [ ${ }^{\circ}$ ], letting the front-left wheel be the lifted leg with the front rolladjustment shaft rotated at an angular velocity $\dot{\theta}_{r f}=0.1$ $[\mathrm{rad} / \mathrm{s}]$ and $\dot{\theta}_{o}$ in the swing phase is $0.1[\mathrm{rad} / \mathrm{s}]$, leg lifting height is $0.15[\mathrm{~m}]$, and distance along the $\mathrm{Y}$ axis in the swing phase is $0.15[\mathrm{~m}]$. These conditions are chosen in order to confirm the degree of errors under the conditions that can be affected by approximation and are within the usable range. Lifting occurred between $0[\mathrm{~s}]$ and $3.7[\mathrm{~s}]$ and landing between $6.5[\mathrm{~s}]$ and $10[\mathrm{~s}]$. Although there is an error of at most $0.007[\mathrm{~m}]$ during lifting and landing, the leg moved up and down while almost maintaining a constant $\mathrm{Y}$ coordinate. As for the $\mathrm{Z}$ coordinate, the leg moves up to 0.15 [m] in height and shifts to the swing phase between $0[\mathrm{~s}]$ and 3.7 [s]. The reason for increase in leg height between 3.7 [s] and $6.5[\mathrm{~s}]$ is the constant position maintained by the front roll-adjustment shaft during the swing phase, which changes the body pitch angle with a change in the front steering angle and causes the leg height to increase. Fig. 11 shows that the desired trajectory is achieved.
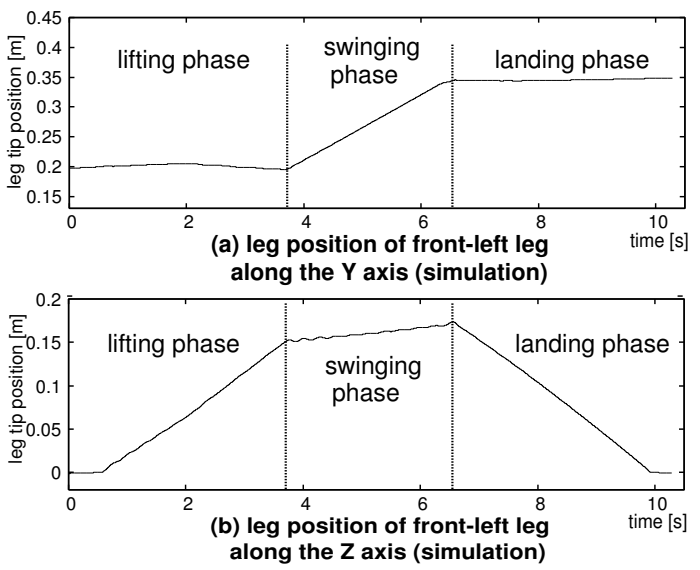

Fig. 11. Leg trajectory (simulation) 


\section{Study of Control Method}

I describe here a control method for the step-up gait shown in Fig. 3 using the equipped sensors. Before and after the step, the robot runs with its wheels using the basic movement control method (Fig.3(a),(1)). If the front wheel touches the step when running, the robot stops and the wheel torque increases, thereby detecting the start of the upward step (b). When detecting the start of the step, the rear steering is rotated such that the margin of static stability during leg motion increases (c). In order to lift a leg, the front rolladjustment shaft is switched from the posture control in equation (1) to the angle control, and the leg is lifted to the desired height. Meanwhile, to prevent the seat part from inclining, the rear roll-adjustment shaft continues to adopt the posture control in equation (1). The pitch adjustment shaft also uses the control in equation (1), and the posture of the sheet is maintained on a horizontal plane. After that, the angle of the front roll-adjustment shaft is kept constant, and the wheel is moved forward while keeping it lifted (d). After moving forward, the wheel is moved down and when landing is detected, leg motion of the front-left wheel ends. As for landing detection, as can be seen in Fig.13(a), since a sign of the roll angle of the seat part changes at around 9 [s] when the wheel lands, this timing can be used for detection.

Next, the front-right wheel becomes an lifted leg (Fig.3(e),(f)). After the front wheels go up the step, by switching to the basic movement control method while adjusting the body rotation relative to the step for ensuring static stability for the rear wheels to go up, the robot moves forward until the rear wheel touches the step $((\mathrm{g}),(\mathrm{h}),(\mathrm{i}))$. The rear wheels are moved closer to the step so that the leg motion period, in which static stability decreases, can be as short as possible. When the rear wheels detect the step, the rear wheels are also lifted onto the step along with the front wheels $((\mathrm{j}),(\mathrm{k}))$.

\section{EVAluation ThROUgh Simulation AND EXPERIMENT}

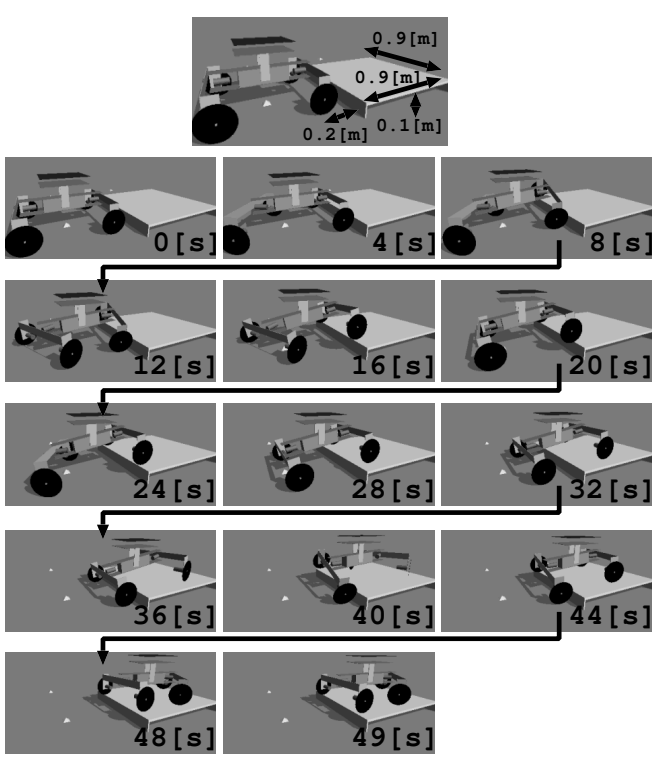

Fig. 12. Simulation of the step-up gait
To evaluate the proposed step-up gait, I performed a simulation and conducted an experiment. The conditions of the experiment were height of the step $0.1[\mathrm{~m}]$; angular velocity of the roll-adjustment shaft when lifting the wheel $\dot{\theta}_{r_{u p}}=0.2[\mathrm{rad} / \mathrm{s}]$; angular velocity of the roll-adjustment shaft when landing the wheel $\dot{\theta}_{r_{\text {down }}}=0.1[\mathrm{rad} / \mathrm{s}]$; angular velocity during the swing phase $\dot{\theta}_{o}=0.2[\mathrm{rad} / \mathrm{s}]$. From the findings on stability, I let the angle of the rear steering shaft when lifting the first rear wheel be $0\left[^{\circ}\right.$ ]. In other words, after the front wheels go up onto the step and the rear wheels move closer to the step, the robot is moved forward adjusting the angle of the rear steering shaft so that it becomes $0\left[{ }^{\circ}\right]$. For detecting a step, I set the threshold values as $3.7[\mathrm{Nm}]$ for the front wheels and as $3.8[\mathrm{Nm}]$ for the rear wheels. The robot was moved in a straight direction of the step when it reached $0.2[\mathrm{~m}]$ before the step.
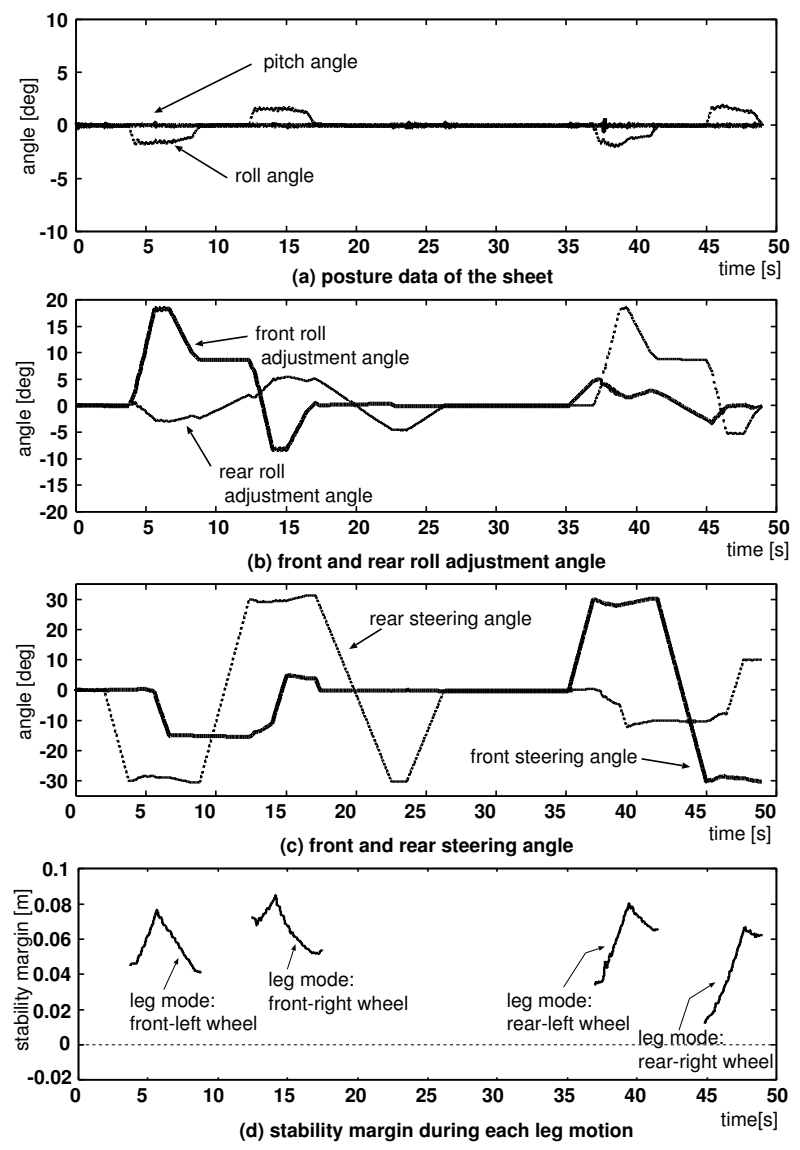

Fig. 13. Simulation data (step-up gait)

Fig.12 depicts the simulation. Fig.13 shows the posture angle of the seat part, angle of the front and rear rolladjustment shafts, front and rear steering angles, and static stability during leg motion.

Fig.13(a) shows that the pitch posture angle of the seat part is almost maintained at horizontal. The roll posture angle of the seat part is inclining approximately $\pm 1.5\left[^{\circ}\right]$ at around 5 [s], 15 [s], 40 [s], and 47 [s] because the twisting force around the body, caused by the roll-adjustment shaft that produced the torque for lifting the wheel, disturbs the posture control of the other roll-adjustment shaft. 
Fig.13(b) shows transition of angles of the front and rear roll-adjustment shafts. From 4 [s] to 9 [s], the front-left wheel is an lifted leg. First, the wheel is lifted until the front rolladjustment shaft is inclined at $18\left[^{\circ}\right]$ (4 [s] to 5.5 [s]). From 5.5 [s] to 7 [s], the front steering is rotated until the angle becomes $-15\left[^{\circ}\right]$ so that the wheel moves forward (Fig.13(c)). Then the wheel moves downward from 7 [s] to 9 [s]. Since the roll angle of the seat part changes from - to + , landing of the wheel can be detected. The other legs behave similarly.

Fig.13(c) shows transition of angles of the front and rear steering shafts. From 2.5 [s] to 17 [s], front wheels are lifted legs. While the front-left wheel is the lifted leg, the rear steering shaft rotates to its steering limit of $-30\left[^{\circ}\right.$ ] so that static stability increases (2.5 [s] to 4.0 [s]). After lifting the front-left wheel, the wheel is moved forward until the front steering shaft is inclined at $-15\left[^{\circ}\right]$ (5.5 [s] to $\left.7.0[\mathrm{~s}]\right)$. While the front-right wheel is the lifted leg, the rear steering shaft is rotated to a steering limit of $30\left[^{\circ}\right]$ so that static stability increases $(9[\mathrm{~s}]$ to $13[\mathrm{~s}])$. The rear steering shaft is rotated to -30 [ $^{\circ}$ ] (17 [s] to 22.5 [s]) after the front wheels are lifted, thereby adjusting the angle of the rear steering shaft to 0 ${ }^{\circ}{ }^{\circ}$ ] when lifting the rear wheels. Rear wheels are lifted legs between 35 [s] and 49 [s]. While the rear-left wheel is the lifted leg, the wheel is moved forward until the rear steering shaft is inclined at $-12\left[^{\circ}\right]$ (38 [s] to 39 [s]). The front steering shaft is rotated to $\pm 30\left[^{\circ}\right]$ in order to ensure static stability. These values were experimentally set for confirming if the robot can move up the step by means of the proposed step-up gait, not using the optimum values.

Fig.13(d) shows the data of static stability only during leg motion, becuase static stability is large enough while four wheels support the body. These data are applicable for a roll angle of $0\left[^{\circ}\right]$ since the inclination of the roll angle of the sheet in (a) can be reduced by adjusting gain. The figure also shows that static stability is maintained.

Fig.14 shows the step-up gait experiment. The conditions of the experiment are the same as the simulation. The real machine could also move up onto the $0.1[\mathrm{~m}]$-high step, and thus, the proposed step-up gait was proven effective. It took about 7 [s] longer than the simulation because the roll angle of the seat part was inclined more than that in the simulation and therefore landing detection of each wheel which used this roll angle was delayed.

\section{CONCLUSION}

In this study, I proposed a “ step-up gait” enabling the mobile robot RT-Mover, which only has 5 driving shafts other than 4 driving wheels, to move up onto a step while maintaining the seat part on a horizontal plane for mounting load by means of a simple four-leg mechanism. I presented that static stability can be maintained during the step-up gait for up to $0.2[\mathrm{~m}]$, and discussed the leg trajectory and control method of each shaft. In addition, I validated by the simulation and experiment that the robot can move up onto a step of $0.1[\mathrm{~m}]$ by means of the proposed gait.

This study only dealt with the case in which the robot goes towards the step in a straight direction. As for the step-

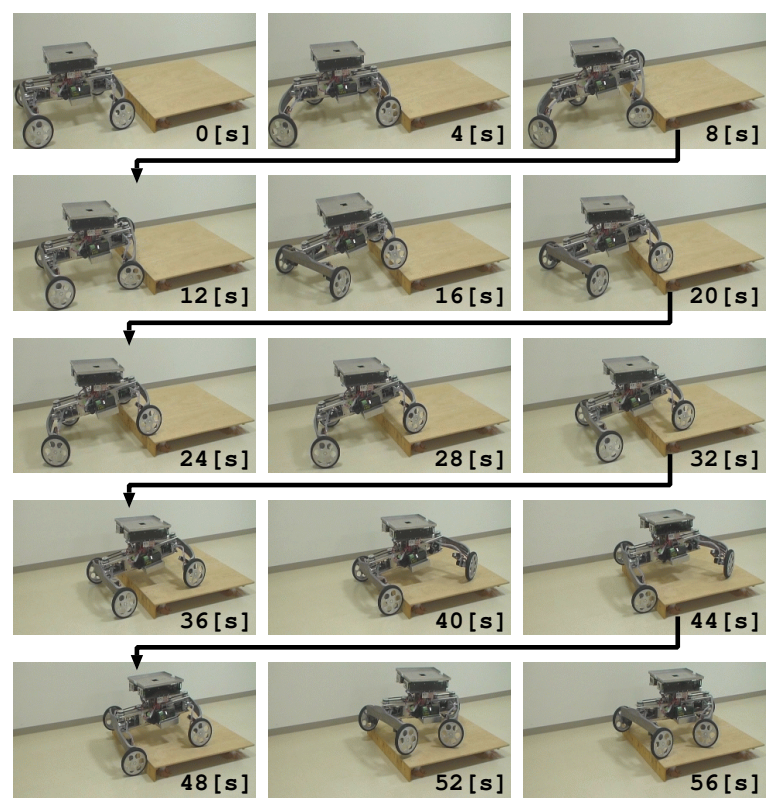

Fig. 14. Experimental scenario (step-up gait)

up gait, I need to study control methods for applying the proposed gait to more general cases, such as when the other part of the road around the steps is uneven or when the robot goes toward the step in an oblique direction.

\section{ACKNOWLEDGEMENT}

This work was supported by Mr. Kazuhisa Ietomi, a master student of our laboratory. I would like to express my gratitude for his support.

\section{REFERENCES}

[1] Shuro Nakajima, "Concept of a Four-wheel-type Mobile Robot for Rough Terrain, RT-Mover", Proceedings of the International Conference on Inteligent Robots and Systems, pp.3257-3264, 2009.

[2] S. M. Song and K. J. Waldron, "Machines That Walk: The Adaptive Suspension Vehicle," MIT Press, 1989.

[3] S. Hirose, et al., "The Gait Control System of the Quadruped Walking Vehicle”, Journal of the Robotics Society of Japan, vol.3, no.4, pp.304 323, 1985.

[4] T. Kubota, Y. Kuroda, Y. Kunii, I. Nakatani, "Small, light-weight rover "Micro5" for lunar exploration ", Acta Astronautica, 52, 447-453, 2003.

[5] R.Volpe, J. Balaram, T. Ohm, R. Ivlev, “Rocky 7: A next generation Mars rover prototype ", Journal of Advanced Robotics, vol.11, no.4, pp.341 358, 1997.

[6] M. Lauria, et al., "Design and control of an innovative microrover", Proceedings of the Fifth ESA Workshop on Advanced Space Technologies for Robotics and Automation, The Netherlands, 1998.

[7] M. V. Winnendael, et al., "Nanokhod micro-rover heading towards Mars”, Proceedings of the Fifth International Symposium on Artificial Intelligence, Robotics and Automation in Space, pp.69-76, 1999.

[8] I. Leppanen, "Workpartner-HUT automations new hybrid walking machine", Proceedings of the CLAWAR' 98, 1998.

[9] S. Hirose and H. Takeuchi, "Roller-Walker: A Proposal of New Leg- wheel Hybrid Mobile Robot", Proceedings of the International Conference on Advanced Robotics, pp.917 922, 1995.

[10] S. Nakajima and E. Nakano, “Adaptive Gait for Large Rough Terrain of a Leg-wheel Robot (Fifth Report: Integrated Gait) ", Journal of Robotics and Mechatronics, vol.21, no.3, pp.419-426, 2009.

[11] K. Six, A. Kecskem "ethy, "Steering properties of a combined wheeled and legged striding excavator", Proceedings of the 10th World Congress on the Theory of Machines and Mechanisms, vol.1, pp.135$140,1999$. 